Open J. Math. Anal., Vol. 2(2018), No. 2, pp. 27 - 40

Website: https://pisrt.org/psr-press/journals/oma/

ISSN: 2616-8111 (Online) 2616-8103 (Print)

http://dx.doi.org/10.30538/psrp-oma2018.0015

\title{
VISCOSITY METHODS FOR APPROXIMATING SOLUTIONS OF VARIATIONAL INEQUALITIES FOR ASYMPTOTICALLY NONEXPANSIVE MAPPINGS
}

\author{
MUHAMMAD AKMAL ${ }^{1}$, MUHAMMAD SAQIB KHAN, SHAHZAD AHMAD MAITLA
}

\begin{abstract}
The aim of this paper is to present a viscosity approximation method for asymptotically nonexpansive mappings in Banach spaces. The strong convergence of the viscosity rules is proved with some assumptions. This paper extend and improve results presented in [1, 2, 3, 4].
\end{abstract}

Mathematics Subject Classification: 47J25, 47N20, 34G20, 65J15.

Key words and phrases: Fixed point; asymptotically nonexpansive mapping; Noor iteration; viscosity approximation; normalized duality mapping.

\section{Introduction}

Special mappings having fixed point, like contractive, non-expansive and asymptotically non-expansive etc, have become a field of interest on their own and have a variety of application in related field like signal processing, image recovery and geometry of objects [5] as well as in IMRT optimization to pre-compute dosedeposition coefficient(DDC) matrix, see [6]. Almost in all branches of mathematics, we see some version of theorems relating to fixed points of functions of special nature. Because of the vast range of applications in almost all areas of everyday life, the research in this field is moving rapidly and an immense literature is present now.

Any equation that can be written as $T(x)=x$, for some map $T$, that is contracting with respect to some (complete) metric on $X$, will provide such a fixed

Received 19-05-218. Revised 14-08-2018. Accepted 18-09-2018.

1 Corresponding Author

(C) 2018 Muhammad Akmal, Muhammad Saqib Khan and Shahzad Ahmad Maitla. This is an open access article distributed under the Creative Commons Attribution License, which permits unrestricted use, distribution, and reproduction in any medium, provided the original work is properly cited. 
point iteration. Mann iteration method, [7, was the stepping stone in this regard and is invariably used in most of the problems. But it only ensures week convergence, see 8 . We require strong convergence in real world problems relating to Hilbert spaces 9. A large amount of research work is dedicated for the modification of Mann process, to control and ensure the strong convergence (see [10, 11, 12, 13, 14, 15, 16]). The first modification of Mann process was proposed by Nakajo et al. in 2003 [10]. They introduced this modification for only one nonexpansive mapping, whereas, Kim et al. introduced a variant for asymptotically nonexpansive mappings, in Hilbert spaces, in the year 2006 [12]. In the same year, Martinez et al. introduced Ishikawa iterative scheme for nonexpansive mappings in Hilbert spaces 13. They gave a variant of Halpern method. $\mathrm{Su}$ et al. in 14 gave a hybrid iteration process for monotone nonexpansive mappings. Liu et al. gave a novel method for quasi-asymptotically finite family of pseudo-contractive mappings [16. Others have also worked on this problem. For more detail, see [17, 18, 19, 20, 21, 22, 23, 24, 25, 26, 27.

In this paper, by using viscosity approximation methods for asymptotically nonexpansive mappings, we obtained fixed point of an iterative sequence, which is the unique solution of variational inequality, with some sufficient and necessary conditions. The results presented in this paper extend and improve mainly results in 28, which primarily are the improvement and extension of results in [1, 2, 3, 4,

\section{Preliminaries}

Throughout this paper, we will assume $E$ to be a real Banach space, with $M \neq \emptyset$ be its closed, bounded and convex subset. Also, $T$ will be a mapping from $M$ to itself and $F(T)$ will denote the set of fixed points of $T$. T is said to be nonexpansive, if for all $y, z \in M,\|T(y)-T(z)\| \leq\|y-z\|$. It will be called asymptotically nonexpansive, if $\exists$ a sequence $l_{m}$ in $[1, \infty)$ with $\lim _{m \rightarrow \infty} l_{m}=1$, such that $\forall y, z \in M$ and $m \geq 0,\left\|T^{m}(y)-T^{m}(z)\right\| \leq l_{m}\|y-z\|$. Similarly, $T$ is called uniformly $L$-Lipschitzian, if $\exists L>0$ such that $\forall y, z \in M$ and $m \geq 0$, $\left\|T^{m}(y)-T^{m}(z)\right\| \leq L\|y-z\|$.

Remark 2.1. Every mapping, which is contractive, is also nonexpansive. Similarly, every mapping, which is nonexpansive, is also asymptotically nonexpansive. Finally, every asymptotically nonexpansive is uniformly $L$-Lipschitzian with appropriate constants. Generally, the converses of these statements do not hold. The asymptotically nonexpansive mappings are important generalization of nonexpansive mappings. For further details, see [29].

Let $f \in E^{*}$, where $E^{*}$ is the dual of $E$. The inner product of $f \in E^{*}$ and $x \in E$, denoted by $\langle f, x\rangle$, is called the duality pairing on $E$. Let $P\left(E^{*}\right)$ denote the power set of $E^{*}$. Define $J: E \rightarrow P\left(E^{*}\right)$, for any $y \in E$, as $J(y)=\{j \in$ $\left.E^{*}:\langle y, j\rangle=\|y\|^{2}=\|j\|^{2}\right\}$. This $J$ is called the normalized duality pairing of $E$. We will use $j$ as the single-valued normalized duality mapping in $J$. 
Let $S$ be a unit sphere, in some Banach space $E$, i-e, $S=\{y \in E:\|y\|=1\}$. $E$ is said to have Gâteaux differentiable norm if, for every $y, z \in S$, the limit $\lim _{h \rightarrow 0} \frac{\|y+h z\|-\|y\|}{h}$ exists. If for each $y \in S$, the limit exists uniformly for $x \in S$, then, $E$ is said to have Gâteaux differentiable norm.

Remark 2.2. It is well-known that, if $E$ has a uniformly Gâteaux differentiable norm, then the normalized duality mapping $J: E \rightarrow P\left(E^{*}\right)$ is uniformly continuous from the norm topology of $E$ to the weak* topology of $E^{*}$ on any bounded subsets of $E$.

The Normal structure coefficient is defined as $N(E)=\inf _{M \subset E}\left\{\frac{d(M)}{r(M)}\right\}$, where $d(M)$ and $r(M)$ are the diameter and Chebyshev radius of $M$, respectively [28. If $N(E)>1$, then, $E$ is said to have uniform normal structure. A space which has uniform normal structure is also known as reflexive.

The variational inequality problem is the problem of solving the inequality, $\langle F(y), z-y\rangle \geq 0$, for some $y \in M$ and $\forall z \in M$. Here, $F: M \rightarrow E^{*}$.

A linear continuous functional, $v \in\left\{l^{\infty}\right\}^{*}$, is called a Banach limit 30, if $\|v\|=1, v_{m}\left(\xi_{m}\right)=v_{m}\left(\xi_{m+1}\right)$ and $\liminf _{m \rightarrow \infty} \xi_{m} \leq v_{m}\left\|\xi_{m}\right\| \leq \limsup _{m \rightarrow \infty} \xi_{m}$. This is true for all $x=\left\{\xi_{i}\right\} \in l^{\infty}$. Here, common notation is to write $v_{m}\left(\xi_{m}\right)$, instead of $v(x)$.

In order to prove our main theorem, we will need the following results.

Lemma 2.3. 21] Let $E$ be a Banach space having uniform normal structure, $M$ a non-empty bounded subset of $E$ and $T: M \longrightarrow M$ be a uniformly L-Lipschitzian mapping with $L<\sqrt{N(E)}$. Suppose also that there exist a nonempty, bounded convex subset $A$ of $M$ with the property that if $x \in A$, the weak $\omega$-limit set of $T$ at $x$, denoted by $\omega_{w}(x)$, is a subset of $A$, i-e, for some $m_{i} \rightarrow \infty$,

$$
\omega_{w}(x):=\left\{y \in E: y=\text { weak }-\lim _{i} T^{m_{i}}(x), x \in A\right\} \subset A,
$$

then, $T$ has a fixed point in $M$.

Lemma 2.4. 31 Let $\left\{\xi_{m}\right\},\left\{\eta_{m}\right\}$ and $\left\{\gamma_{m}\right\}$ be three non-negative real sequences, with $\eta_{m}=o\left(\left\{\xi_{m}\right\}\right), \sum_{m=0}^{\infty} \gamma_{m}<\infty$ and $\xi_{m+1} \leq\left\{1-\mu_{m}\right\} \xi_{m}+\eta_{m}+\gamma_{m}, \forall m \geq m_{0}$, $m_{0} \in \mathbb{Z}^{+}$, where $\left\{\mu_{m}\right\} \subset(0,1)$, with $\sum_{m=0}^{\infty} \mu_{m}=\infty$. Then, $\lim _{m \rightarrow \infty} \xi_{m}=0$.

Lemma 2.5. 23. Let $E$ be a real Banach space and $J$ be a normalized duality mapping on $E$. For any $y, z \in E, j(y+z) \in J(y+z)$ and $j(y) \in J(y)$, the following statements are true.

(1) $\|y+z\|^{2} \leq\|y\|^{2}+2\langle z, j(y+z)\rangle$,

(2) $\|y+z\|^{2} \geq\|y\|^{2}+2\langle z, j(y)\rangle$. 
Lemma 2.6. 28. Let $\left(t_{m}\right)$ be a sequence in $(0,1)$, such that $\lim _{m \rightarrow \infty} t_{m}=1$. Also, let $\left(l_{m}\right)$ be a sequence in $[1, \infty)$ with $\lim _{m \rightarrow \infty} l_{m}=1$. Then, for any $\alpha \in(0,1)$, the following are true $\forall m \geq 0$

(1) $0<t_{m}<\frac{(1-\alpha) l_{m}}{l_{m}-\alpha}$

(2) $\left(l_{m}^{2}-1\right)<\left(1-\frac{t_{m}}{l_{m}}\right)^{2}$

(3) $\frac{l_{m}-1}{l_{m}-t_{m}}<\frac{l_{m}-t_{m}}{\left(l_{m}+1\right) l_{m}^{2}} \rightarrow 0$.

\section{Main Result}

We have a well known Noor iterative process 32. If $\left(\alpha_{m}\right),\left(\beta_{m}\right)$ and $\left(\gamma_{m}\right)$ are sequences in $[0,1]$, then,

$$
\begin{aligned}
x_{m+1} & =\alpha_{m} x_{m}+\left(1-\alpha_{m}\right) T\left(y_{m}\right) \\
y_{m} & =\beta_{m} x_{m}+\left(1-\beta_{m}\right) T\left(x_{m}\right) \\
z_{m} & =\gamma_{m} x_{m}+\left(1-\gamma_{m}\right) T\left(x_{m}\right) .
\end{aligned}
$$

Corresponding to above, we have following three step viscosity approximation method for asymptotically nonexpansive mappings in Banach spaces, whose strong convergence is also proved below.

Theorem 3.1. Let $E$ be a real Banach space and let the norm on $E$ be uniformly Gâteaux differentiable, possessing uniform normal structure. Let $M \neq \emptyset$ be bounded, closed and convex subset of $E$ and let $k: M \rightarrow M$ be a contraction with $\alpha \in(0,1)$ be its contractive constant. Also, let $T: M \rightarrow M$ be asymptotically nonexpansive mapping, $\left(l_{m}\right)$ a sequence in $[1, \infty)$, such that $\sum_{m=0}^{\infty}\left(l_{m}-1\right)<\infty$ and $\lim _{m \rightarrow \infty} l_{m}=1$. Also, let $\left(t_{m}\right)$ be a sequence in $(0,1)$ such that $\forall m \geq 0$, $t_{m} \in\left(0, \eta_{m}\right)$, where $\eta_{m}=\left\{\frac{(1-\alpha) l_{m}}{l_{m}-\alpha}, l_{m}\left(1-\sqrt{l_{m}^{2}-1}\right)\right\}$ and $\lim _{m \rightarrow \infty} t_{m}=1$.

Given any $x_{0} \in M$ and sequences $\left(\alpha_{m}\right),\left(\beta_{m}\right)$ and $\left(\gamma_{m}\right)$ in $[0,1]$, define a sequence $\left(x_{m}\right)$ as follows.

$$
\begin{aligned}
x_{m+1} & =\alpha_{m} k\left(x_{m}\right)+\left(1-\alpha_{m}\right) T^{m}\left(y_{m}\right) \\
y_{m} & =\beta_{m} x_{m}+\left(1-\beta_{m}\right) T^{m}\left(z_{m}\right) \\
z_{m} & =\gamma_{m} x_{m}+\left(1-\gamma_{m}\right) T^{m}\left(x_{m}\right) .
\end{aligned}
$$

Then, for every $m \geq 0, \exists N_{m} \in M$, such that

$$
N_{m}=\left\{1-\frac{t_{m}}{l_{m}}\right\} k\left(x_{m}\right)+\frac{t_{m}}{l_{m}} T^{m}\left(N_{m}\right) .
$$

Also, $\left(N_{m}\right)$ and $\left(x_{m}\right)$ are strongly convergent to some $h \in F(T)$ which, $\forall u \in$ $F(T)$, is the unique solution of the variational inequality $\langle(I-k) h, j(h-u)\rangle \leq 0$, if and only if the following conditions hold. 
(1) $\lim _{m \rightarrow \infty}\left\|N_{m}-T\left(N_{m}\right)\right\|=0$ and $\lim _{m \rightarrow \infty}\left\|x_{m}-T\left(x_{m}\right)\right\|=0$,

(2) As $m \rightarrow \infty, \alpha_{m} \rightarrow 0$ and $\sum_{m=0}^{\infty} \alpha_{m}=\infty$.

Proof. It follows from Lemma 2.6 that for each $m \geq 0, t_{m} \in\left\{0, \frac{(1-\alpha) l_{m}}{l_{m}-\alpha}\right\}$. Define $U_{m}: M \rightarrow M$ as,

$$
U_{m}(x)=\left(1-\frac{t_{m}}{l_{m}}\right) k(x)+\frac{t_{m}}{l_{m}} T^{m}(x) .
$$

It can easily be checked that $U_{m}$ is a contraction. By Banach fixed point theorem, there exists a unique fixed point $N_{m} \in M, \forall m \geq 0$, such that

$$
U_{m}\left(N_{m}\right)=\left(1-\frac{t_{m}}{l_{m}}\right) k\left(N_{m}\right)+\frac{t_{m}}{l_{m}} T^{m}\left(N_{m}\right)=N_{m} .
$$

Next, we want to show that $\left(N_{m}\right)$ is a sequence that converges strongly to some $h \in F(T)$, which is the unique solution of variational inequality $\langle(I-k) h, j(h-$ $u)\rangle \leq 0$. To show this, let $h \in F(T)$. Then, using lemma 2.5, we get,

$$
\begin{aligned}
\left\|N_{m}-h\right\|^{2} \leq & 2\left\langle N_{m}-k\left(N_{m}\right), j\left(N_{m}-h\right)\right\rangle+2\left\langle k\left(N_{m}\right)-k(h), j\left(N_{m}-h\right)\right\rangle \\
& +2\left\langle k(h)-h, j\left(N_{m}-h\right)\right\rangle \\
\leq & 2\left\langle N_{m}-k\left(N_{m}\right), j\left(N_{m}-h\right)+\left\{\alpha^{2}+2 \alpha\right\}\left\|N_{m}-h\right\|^{2}\right. \\
& +2\left\langle k(h)-h, j\left(N_{m}-h\right)\right\rangle .
\end{aligned}
$$

This means that

$$
\begin{aligned}
\left(1-\alpha^{2}-2 \alpha\right)\left\|N_{m}-h\right\|^{2} \leq & 2\left\langle N_{m}-k\left(N_{m}\right), j\left(N_{m}-h\right)\right\rangle \\
& +2\left\langle k(h)-h, j\left(N_{m}-h\right)\right\rangle .
\end{aligned}
$$

For the second term on the right in (3), define $\psi: M \longrightarrow \mathbb{R}$ by $\psi(x)=\nu_{m} \| N_{m}-$ $x \|^{2}$. Since, $E$ is Banach space having uniform normal structure, it is reflexive. Also, since $\lim _{m \rightarrow \infty} \psi(x)=\infty, \psi$ is continuous and convex. By Schauder Theorem, there exist $x^{\prime} \in M$, such that $\psi\left(x^{\prime}\right)=\inf _{x \in M} \psi(x)$ and the set $A=\{y \in M$ : $\left.\psi(y)=\inf _{x \in M} \psi(x)\right\} \neq \emptyset$. It is also closed, bounded and convex. Since, $\| N_{m}-$ $T\left(N_{m}\right) \| \rightarrow 0$, by assumption, it can readily be seen that $\bigcup_{x \in M} \omega_{w}(x) \subset A$. By Lemma 2.3, $T$ has a fixed point $h \in A$.

Since, $M$ is convex, for any $x \in M$ and $t \in[0,1]$, we have $(1-t) h+t x \in M$. As established above, $\psi$ is continuous, we get $\psi(h) \leq \psi((1-t) h+t x)$. This, with lemma 2.5 . (1), can be written as

$$
\begin{aligned}
0 & \leq \frac{\psi((1-t) h+t x)-\psi(h)}{t} \\
& =\frac{\nu_{m}}{t}\left(\left\|\left(N_{m}-h\right)+\{h-x\} t\right\|^{2}-\left\|N_{m}-h\right\|^{2}\right) \\
& \leq \frac{\nu_{m}}{t}\left(\left\{\left\|N_{m}-h\right\|^{2}+2\left\langle(h-x) t, j\left(N_{m}-h+t(h-x)\right)\right\rangle\right\}-\left\|N_{m}-h\right\|^{2}\right)
\end{aligned}
$$




$$
=2 \nu_{m}\left\langle h-x, j\left(N_{m}-h+t(h-x)\right)\right\rangle .
$$

This implies that

$$
2 \nu_{m}\left\langle x-h, j\left(N_{m}-h+t(h-x)\right)\right\rangle \leq 0 .
$$

Since, $M$ is bounded and $j$ is norm-to-weak uniformly continuous, letting $t \rightarrow 0$ $\forall x \in M$, we have

$$
2 \nu_{m}\left\langle x-h, j\left(N_{m}-h\right)\right\rangle \leq 0 .
$$

In particular, since $h \in M$ and $k: M \rightarrow M, \exists x \in M$ such that $k(h)=x$. Thus, the above equation can be written as

$$
2 \nu_{m}\left\langle k(h)-h, j\left(N_{m}-h\right)\right\rangle \leq 0 .
$$

For the first term on the right in (3), $\forall u \in F(T)$, we can write $\left\langle N_{m}-\right.$ $\left.k\left(N_{m}\right), j\left(N_{m}-u\right)\right\rangle$ by using (2), as follows:

$$
\begin{aligned}
& \left\langle N_{m}-k\left(N_{m}\right), j\left(N_{m}-u\right)\right\rangle \\
= & \left\langle\left(1-\frac{t_{m}}{l_{m}}\right) k\left(N_{m}\right)+\frac{t_{m}}{l_{m}} T^{m}\left(N_{m}\right)-k\left(N_{m}\right), j\left(N_{m}-u\right)\right\rangle \\
= & \frac{t_{m}}{l_{m}}\left\langle N_{m}-k\left(N_{m}\right), j\left(N_{m}-u\right)\right\rangle \\
& +\frac{t_{m}}{l_{m}}\left\langle T^{m}\left(N_{m}\right)-N_{m}, j\left(N_{m}-u\right)\right\rangle .
\end{aligned}
$$

This implies that

$$
\left\langle N_{m}-k\left(N_{m}\right), j\left(N_{m}-u\right)\right\rangle=\frac{t_{m}}{l_{m}-t_{m}}\left\langle T^{m}\left(N_{m}\right)-N_{m}, j\left(N_{m}-u\right)\right\rangle .
$$

Note that,

$$
\left\langle N_{m}-T^{m}\left(N_{m}\right), j\left(N_{m}-u\right)\right\rangle=\left\|N_{m}-u\right\|^{2}-\left\langle T^{m}\left(N_{m}\right)-u, j\left(N_{m}-u\right)\right\rangle .
$$

By lemma 2.5 2, we have

$$
\left\|N_{m}-u\right\|^{2}-\left\|N_{m}-u+T^{m}\left(N_{m}\right)-u\right\|^{2} \leq-2\left\langle T^{m}\left(N_{m}\right)-u, j\left(N_{m}-u\right)\right\rangle .
$$

Also,

$$
\begin{aligned}
\left\|\left\{N_{m}-u\right\}+\left\{T^{m}\left(N_{m}\right)-u\right\}\right\|^{2} \leq & \left\|N_{m}-u\right\|^{2}+\left\|T^{m}\left(N_{m}\right)-u\right\|^{2} \\
& +2\left\|N_{m}-u\right\|\left\|T^{m}\left(N_{m}\right)-u\right\| \\
\leq & \left\{1+l_{m}^{2}+2 l_{m}\right\}\left\|N_{m}-u\right\|^{2} \\
= & \left\{l_{m}+1\right\}^{2}\left\|N_{m}-u\right\|^{2} .
\end{aligned}
$$

This implies that

$$
\begin{aligned}
-l_{m}\left\{l_{m}+2\right\}\left\|N_{m}-u\right\|^{2} & \leq\left\|N_{m}-u\right\|^{2}-\left\|\left\{N_{m}-u\right\}+\left\{T^{m}\left(N_{m}\right)-u\right\}\right\|^{2} \\
& \leq-2\left\langle T^{m}\left(N_{m}\right)-u, j\left(N_{m}-u\right)\right\rangle .
\end{aligned}
$$

This means that

$$
-\frac{l_{m}}{2}\left\{l_{m}+2\right\}\left\|N_{m}-u\right\|^{2} \leq-\left\langle T^{m}\left(N_{m}\right)-u, j\left(N_{m}-u\right)\right\rangle .
$$


Thus, our equation becomes

$$
\begin{aligned}
\left\{\frac{2-l_{m}^{2}-2 l_{m}}{2}\right\}\left\|N_{m}-u\right\|^{2} & =\left\|N_{m}-u\right\|^{2}-\frac{l_{m}}{2}\left\{l_{m}+2\right\}\left\|N_{m}-u\right\|^{2} \\
& \leq\left\|N_{m}-u\right\|^{2}-\left\langle T^{m}\left(N_{m}\right)-u, j\left(N_{m}-u\right)\right\rangle \\
& =\left\langle N_{m}-T^{m}\left(N_{m}\right), j\left(N_{m}-u\right) .\right\rangle
\end{aligned}
$$

The above equation can be written as

$$
\left\langle T^{m}\left(N_{m}\right)-N_{m}, j\left(N_{m}-u\right)\right\rangle \leq\left\{\frac{l_{m}^{2}+2 l_{m}-2}{2}\right\}\left\|N_{m}-u\right\|^{2} .
$$

This means that

$$
\left\langle N_{m}-k\left(N_{m}\right), j\left(N_{m}-u\right)\right\rangle \leq\left\{\frac{t_{m}}{l_{m}-t_{m}}\right\}\left\{\frac{l_{m}^{2}+2 l_{m}-2}{2}\right\}\left\|N_{m}-u\right\|^{2} .
$$

Note that $\frac{l_{m}^{2}+2 l_{m}-2}{2} \rightarrow \frac{1}{2}$ as $m \rightarrow \infty$. Similarly, since $t_{m}>0$ and $l_{m} \geq 1$, for all $m>0, \frac{t_{m}}{l_{m}-t_{m}}>0$. Thus, $\lim _{m \rightarrow \infty} \frac{t_{m}}{l_{m}-t_{m}} \geq 0$. Since, $M$ is bounded, so $\left\|N_{m}-u\right\|$ is bounded, $\forall u \in F(T)$. This shows that

$$
\limsup _{m \longrightarrow \infty}\left\langle N_{m}-k\left(N_{m}\right), j\left(N_{m}-u\right)\right\rangle \leq 0 .
$$

Since, $h \in F(T)$ and (5) is true for all $u \in F(T)$, we have

$$
\limsup _{m \longrightarrow \infty}\left\langle N_{m}-k\left(N_{m}\right), j\left(N_{m}-h\right)\right\rangle \leq 0 .
$$

Using (4) and (6), it can be seen from $\sqrt{3}$. that, $\lim _{m \rightarrow \infty}\left\|N_{m}-h\right\|^{2}=0$. Therefore, there is a subsequence $\left\{N_{m_{c}}\right\} \subset\left\{N_{m}\right\}$, such that $N_{m_{c}} \rightarrow h$, as $c \rightarrow \infty$.

For uniqueness of the $h$, Suppose $\exists$ another subsequence $\left\{N_{m_{i}}\right\} \subset\left(N_{m}\right)$, such that $\lim _{i \rightarrow \infty} N_{m_{i}} \rightarrow s$, where $s \in F(T)$. Since $N_{m_{c}} \rightarrow h$, taking $u=s$ in (5), we get $\langle h-k(h), j(h-s)\rangle \leq 0$. Similarly, since $N_{m_{i}} \rightarrow s$, taking $u=h$ in (5), we get $\langle s-k(s), j(s-h)\rangle \leq 0$. Adding these two gives,

$$
\langle h-s-k(h)+k(s), j(h-s)\rangle \leq 0 .
$$

Therefore we have

$$
\|h-s\|^{2} \leq\left\langle k(h)-h(s), j(h-s) \leq \alpha\|h-s\|^{2} .\right.
$$

Since $\alpha<1$, this implies that $h=s$. Thus, $N_{m} \rightarrow h$ and $h \in F(T)$ is unique. From (5), for all $u \in F(T)$, we have,

$$
\langle h-k(h), j(h-u)\rangle \leq 0 .
$$

Hence $h \in F(T)$ is the unique solution of the variational inequality,

$$
\langle(I-k) h, j(h-u)\rangle \leq 0 .
$$


In order to show that $x_{m}$ converges strongly to $h$, we first need to show that $\limsup _{m \rightarrow \infty}\left\langle k(h)-h, j\left(x_{m+1}-h\right)\right\rangle \leq 0$. For simplicity, let $C_{r}=\frac{t_{r}}{l_{r}}$, for each $r \geq 0$. By using (1) and (2), for any $m, r \geq 0$, we can write

$$
x_{m}-N_{r}=\left(1-C_{r}\right)\left\{x_{m}-k\left(N_{r}\right)\right\}+C_{r}\left\{x_{m}-T^{r}\left(N_{r}\right)\right\} .
$$

Rearranging the above equation gives

$$
C_{r}\left(x_{m}-T^{r}\left(N_{r}\right)\right)=x_{m}-N_{r}-\left(1-C_{r}\right)\left(x_{m}-k\left(N_{r}\right)\right) .
$$

Taking squared norm on both sides and using Lemma 2.6(2) gives

$$
\begin{aligned}
& C_{r}^{2}\left\|x_{m}-T^{r}\left(N_{r}\right)\right\|^{2} \\
\geq & \left\|x_{m}-N_{r}\right\|^{2}-2\left(1-C_{r}\right)\left\langle x_{m}-k\left(N_{r}\right), j\left(x_{m}-N_{r}\right)\right\rangle \\
\geq & \left\|x_{m}-N_{r}\right\|^{2}-2\left(1-C_{r}\right)\left\|x_{m}-N_{r}\right\|^{2}+2\left(1-C_{r}\right)\left\langle k\left(N_{r}\right)-N_{r}, j\left(x_{m}-N_{r}\right)\right\rangle \\
= & \left\{2 C_{r}-1\right\}\left\|x_{m}-N_{r}\right\|^{2}+2\left(1-C_{r}\right)\left\langle k\left(N_{r}\right)-N_{r}, j\left(x_{m}-N_{r}\right)\right\rangle .
\end{aligned}
$$

The rearrangement of the above inequality gives

$$
\begin{aligned}
& \left\langle k\left(N_{r}\right)-N_{r}, j\left(x_{m}-N_{r}\right)\right\rangle \\
\leq & \frac{\left\{1-2 C_{r}\right\}\left\|x_{m}-N_{r}\right\|^{2}+C_{r}^{2}\left\|x_{m}-T^{r}\left(N_{r}\right)\right\|^{2}}{2\left(1-C_{r}\right)} \\
= & \frac{2 C_{r}-1}{2\left(1-C_{r}\right)}\left\{\left\|T^{r}\left(N_{r}\right)-x_{m}\right\|^{2}-\left\|x_{m}-N_{r}\right\|^{2}\right\}+\frac{\left(C_{r}-1\right)^{2}}{2\left(1-C_{r}\right)}\left\|T^{r}\left(N_{r}\right)-x_{m}\right\|^{2} \\
\leq & \frac{2 C_{r}-1}{2\left(1-C_{r}\right)}\left\{\left(\left\|T^{r}\left(N_{r}\right)-T^{r}\left(x_{m}\right)\right\|+\left\|T^{r}\left(x_{m}\right)-x_{m}\right\|\right)^{2}-\left\|x_{m}-N_{r}\right\|^{2}\right\} \\
& +\frac{\left(C_{r}-1\right)^{2}}{2\left(1-C_{r}\right)}\left\|T^{r}\left(N_{r}\right)-x_{m}\right\|^{2} \\
\leq & \frac{2 C_{r}-1}{2\left(1-C_{r}\right)}\left\{\left(l_{r}^{2}-1\right)\left\|N_{r}-x_{m}\right\|^{2}+\left\|T^{r}\left(x_{m}\right)-x_{m}\right\|^{2}+2 l_{r}\left\|N_{r}-x_{m}\right\|\right. \\
& \left.\times\left\|T^{r}\left(x_{m}\right)-x_{m}\right\|\right\}+\frac{\left(C_{r}-1\right)^{2}}{2\left(1-C_{r}\right)}\left\|T^{r}\left(N_{r}\right)-x_{m}\right\|^{2} .
\end{aligned}
$$

Since $\left(x_{m}\right)$ and $\left(N_{r}\right)$ are sequences in $M$, they are bounded. Similarly, since $T: M \rightarrow M,\left\|T^{r}\left(N_{r}\right)-x_{m}\right\|$ is also bounded. So, Let

$G_{1}=\sup _{m, r \geq 0}\left\{\left\|T^{r}\left(N_{r}\right)-x_{m}\right\|,\left\|T^{r}\left(N_{r}\right)-x_{m}\right\|^{2},\left\|N_{r}-x_{m}\right\|,\left\|N_{r}-x_{m}\right\|^{2},\left\|x_{m}-h\right\|\right\}$.

Note that $G_{1}<\infty$. Hence, (7) can be written as,

$$
\begin{array}{r}
\left\langle k\left(N_{r}\right)-N_{r}, j\left(x_{m}-N_{r}\right)\right\rangle \leq \frac{2 C_{r}-1}{2\left(1-C_{r}\right)}\left\{\left(l_{r}^{2}-1\right) G_{1}\right. \\
\left.+2 l_{r} G_{1}\left\|T^{r}\left(x_{m}\right)-x_{m}\right\|+\left\|T^{r}\left(x_{m}\right)-x_{m}\right\|^{2}\right\}+\frac{\left(C_{r}-1\right)^{2}}{2\left(1-C_{r}\right)} G_{1} .
\end{array}
$$


It follows from Lemma 2.6 $(2)$ that $\left(l_{r}^{2}-1\right)<\left(1-C_{r}\right)^{2}$, which shows that,

$$
\frac{\left(2 C_{r}-1\right)}{2\left(1-C_{r}\right)}\left(l_{r}^{2}-1\right) \leq \frac{\left(2 C_{r}-1\right)}{2\left(1-C_{r}\right)}\left(1-C_{r}\right)^{2} \leq \frac{\left(2 C_{r}-1\right)}{2}\left(1-C_{r}\right) .
$$

Substituting (9) in (8), we get,

$$
\begin{aligned}
\left\langle k\left(N_{r}\right)-N_{r}, j\left(x_{m}-N_{r}\right)\right\rangle \leq & C_{r}\left\{1-C_{r}\right\} G_{1} \\
& +\frac{2 C_{r}-1}{2\left(1-C_{r}\right)}\left\{2 l_{r} G_{1}\left\|T^{r}\left(x_{m}\right)-x_{m}\right\|\right. \\
& \left.+\left\|T^{r}\left(x_{m}\right)-x_{m}\right\|^{2}\right\} .
\end{aligned}
$$

Further, by the assumption that $\lim _{m \rightarrow \infty}\left\|x_{m}-T\left(x_{m}\right)\right\|=0$. Hence, for any $r \geq 1$,

$$
\left\|T^{r}\left(x_{m}\right)-x_{m}\right\| \leq\left\{l_{r-1}+l_{r-2}+\ldots+l_{2}+l_{1}\right\}\left\|T\left(x_{m}\right)-x_{m}\right\| \rightarrow 0 .
$$

as $m \rightarrow \infty$. From 10 and 11 ,,$\forall r \geq 0$, we have

$$
\limsup _{m \rightarrow \infty}\left\langle k\left(N_{r}\right)-N_{r}, j\left(x_{m}-N_{r}\right\rangle \leq C_{r}\left\{1-C_{r}\right\} G_{1} .\right.
$$

Since, $\lim _{r \rightarrow \infty} t_{r}=1$ and $\lim _{r \rightarrow \infty} l_{r}=1$, we have, $\lim _{r \rightarrow \infty} C_{r}=1$. Thus,

$$
\limsup _{r \rightarrow \infty} \limsup _{m \rightarrow \infty}\left\langle k\left(N_{r}\right)-N_{r}, j\left(x_{m}-N_{r}\right\rangle \leq 0 .\right.
$$

Since, $N_{r} \rightarrow h \in F(T)$ and $k$ is a contraction map, $k\left(N_{r}\right) \rightarrow k(h)$. Also, $J$ is uniformly continuous from the norm topology of $E$ to weak ${ }^{*}$ topology of $E^{*}$ on any bounded subset of $E$, hence for any given $\varepsilon>0, \exists$ a positive integer $m_{0}$, such that for any $m, r \geq m_{0}$ we have

$$
\begin{aligned}
\left|\left\langle h-N_{r}, j\left(x_{m}-N_{r}\right)\right\rangle\right| & <\frac{\epsilon}{3} \\
\mid\left\langle k\left(N_{r}\right)-k(h), j\left(x_{m}-N_{r}\right\rangle\right| & <\frac{\varepsilon}{3} \\
\left|\left\langle k(h)-h, j\left(x_{m}-N_{r}\right)-j\left(x_{m}\right)-h\right\rangle\right| & <\frac{\varepsilon}{3} .
\end{aligned}
$$

Hence, for any $m, r \geq m_{0}$ we have

$$
\begin{aligned}
& \left|\left\langle k\left(N_{r}\right)-N_{r}, j\left(x_{m}-N_{r}\right)\right\rangle-\left\langle k(h)-h, j\left(x_{m}\right)-h\right\rangle\right| \\
\leq & \left|\left\langle k\left(N_{r}\right)-k(h), j\left(x_{m}-N_{r}\right)\right\rangle\right|+\left|\left\langle k(h)-h, j\left(x_{m}-h\right)\right\rangle\right| \\
& +\left|\left\langle h-N_{r}, j\left(x_{m}-N_{r}\right)\right\rangle\right| \\
< & \frac{\varepsilon}{3}+\frac{\varepsilon}{3}+\frac{\varepsilon}{3}=\varepsilon .
\end{aligned}
$$

From $(12)$ and 13 , we have,

$$
\begin{aligned}
& \limsup _{m \rightarrow \infty}\left\langle k(h)-h, j\left(x_{m}-h\right)\right\rangle \\
& \leq \limsup _{r \rightarrow \infty} \limsup _{m \rightarrow \infty}\left\langle k\left(N_{r}\right)-N_{r}, j\left(x_{m}-N_{r}\right)\right\rangle+\varepsilon \leq \varepsilon .
\end{aligned}
$$

By arbitrariness of $\varepsilon>0$, this becomes,

$$
\limsup _{m \rightarrow \infty}\left\langle k(h)-h, j\left(x_{m}-h\right)\right\rangle \leq 0 .
$$


In order to prove that $x_{m} \rightarrow h$, consider the following.

$$
\begin{aligned}
\left\|x_{m+1}-h\right\|^{2} \leq & \left(1-\alpha_{m}\right)^{2}\left\|T^{m}\left(y_{m}\right)-h\right\|^{2}+2 \alpha_{m}\left\langle k\left(x_{m}\right)-h, j\left(x_{m+1}-h\right)\right\rangle \\
\leq & \left(1-\alpha_{m}\right)^{2} l_{m}^{2}\left\|y_{m}-h\right\|^{2}+2 \alpha_{m}\left\langle k\left(x_{m}\right)-k(h), j\left(x_{m+1}-h\right)\right\rangle \\
& +2 \alpha_{m}\left\langle k(h)-h, j\left(x_{m+1}-h\right)\right\rangle \\
\leq & \left(1-\alpha_{m}\right)^{2} l_{m}^{2}\left\|y_{m}-h\right\|^{2}+2 \alpha_{m} \alpha\left\|x_{m}-h\right\| \cdot\left\|x_{m+1}-h\right\| \\
& +2 \alpha_{m}\left\langle k(h)-h, j\left(x_{m+1}-h\right)\right\rangle .
\end{aligned}
$$

Using (1), we have $\left\|z_{m}-h\right\| \leq l_{m}\left\|x_{m}-h\right\|$ and $\left\|y_{m}-h\right\| \leq \beta_{m}\left\|x_{m}-h\right\|+(1-$ $\left.\beta_{m}\right) l_{m}\left\|z_{m}-h\right\|$. This gives us,

$$
\left\|y_{m}-h\right\| \leq \beta_{m}\left\|x_{m}-h\right\|+\left(1-\beta_{m}\right) l_{m}^{2}\left\|x_{m}-h\right\| \leq l_{m}^{2}\left\|x_{m}-h\right\| .
$$

Now consider the second term of (14)

$$
2 \alpha_{m} \alpha\left\|x_{m}-h\right\| .\left\|x_{m+1}-h\right\| \leq \alpha_{m} \alpha\left\{\left\|x_{m}-h\right\|^{2}+\left\|x_{m+1}-h\right\|^{2}\right\}
$$

Putting the value from (15) and (16) into (14) and solve it.

$$
\begin{array}{r}
\left\|x_{m+1}-h\right\|^{2} \leq\left(1-\alpha_{m}\right)^{2} l_{m}^{6}\left\|x_{m}-h\right\|^{2}+\alpha_{m} \alpha\left\|x_{m}-h\right\|^{2}+\alpha_{m} \alpha\left\|x_{m+1}-h\right\|^{2} \\
+2 \alpha_{m}\left\langle k(h)-h, j\left(x_{m+1}-h\right)\right\rangle .
\end{array}
$$

If we define $d_{m+1}:=\max _{m \geq 0}\left\langle k(h)-h, j\left(x_{m}-h\right)\right\rangle \geq 0$, the above equation can be written as,

$$
\begin{aligned}
& \left(1-\alpha_{m} \alpha\right)\left\|x_{m+1}-h\right\|^{2} \\
& \leq\left(1-\alpha_{m}\right)^{2} l_{m}^{6}\left\|x_{m}-h\right\|^{2}+\alpha_{m} \alpha\left\|x_{m}-h\right\|^{2}+2 \alpha_{m} d_{m+1} \\
& =\left(1-\alpha_{m}\right)^{2}\left(l_{m}^{6}-1\right)\left\|x_{m}-h\right\|^{2}+\left(1-\alpha_{m}\right)^{2} \\
& +\alpha_{m} \alpha\left\|x_{m}-h\right\|^{2}+2 \alpha_{m} d_{m+1} \\
& =\left(1-\alpha_{m}\right)^{2}\left(l_{m}-1\right)\left(l_{m}^{5}+l_{m}^{4}+l_{m}^{3}+l_{m}^{2}+l_{m}+1\right)\left\|x_{m}-h\right\|^{2} \\
& +\left(1-\alpha_{m}\right)\{2-\alpha\}\left\|x_{m}-h\right\|^{2}+\alpha_{m}^{2}\left\|x_{m}-h\right\|^{2}+2 \alpha_{m} d_{m+1} .
\end{aligned}
$$

If we define $G_{2}:=\sup _{m \geq 1}\left\{\left\{l_{m}^{5}+l_{m}^{4}+l_{m}^{3}+l_{m}^{2}+l_{m}+1\right\}\left\|x_{m}-h\right\|^{2}\right\}$, we can write the above equation as

$$
\begin{aligned}
\left\|x_{m+1}-h\right\|^{2} \leq & \frac{\left\{1-\alpha_{m}\right\}^{2}}{1-\alpha_{m} \alpha}\left\{l_{m}-1\right\} G_{2}+\frac{1-\alpha_{m}\{2-\alpha\}}{1-\alpha_{m} \alpha} G_{2} \\
& +\frac{\alpha_{m}^{2}}{1-\alpha_{m} \alpha} G_{2}+\frac{2 \alpha_{m}}{1-\alpha_{m} \alpha} d_{m+1} .
\end{aligned}
$$

By assumption, $\lim _{m \rightarrow \infty} \alpha_{m}=0$. Thus, $\exists m_{0} \in \mathbb{Z}^{+}$, such that $1-\alpha_{m} \alpha>\frac{1}{2}$. This means that

$$
\frac{1-\alpha_{m}\{2-\alpha\}}{1-\alpha_{m} \alpha}=1-\frac{2 \alpha_{m}\{1-\alpha\}}{1-\alpha_{m} \alpha} \leq 1-2 \alpha_{m}\{1-\alpha\} .
$$

Thus, inequality (17) is equivalent to,

$$
\left\|x_{m+1}-h\right\|^{2} \leq 2\left\{1-\alpha_{m}\right\}^{2}\left\{l_{m}-1\right\} G_{2}+\left\{1-2 \alpha_{m}\{1-\alpha\}\right\} G_{2}+2 \alpha_{m}^{2} G_{2}
$$




$$
+\frac{2 \alpha_{m}}{1-\alpha_{m} \alpha} d_{m+1}
$$

Note that, $d_{m} \rightarrow 0$, 28]. It is not difficult to show that the assumptions of lemma 2.4 will be satisfied, if we assume $\xi_{m}=\left\|x_{m}-h\right\|^{2}, \mu_{m}=2\{1-\alpha\} \alpha_{m}$, $\eta_{m}=2 G_{2} \alpha_{m}^{2}+\frac{2 \alpha_{m}}{1-\alpha_{m} \alpha} d_{m+1}$ and $\gamma_{m}=2\left\{1-\alpha_{m}\right\}^{2}\left\{l_{m}-1\right\} G_{2}$. Thus, by lemma $2.4 \lim _{m \rightarrow \infty}\left\|x_{m}-h\right\|^{2}=0$.

Suppose that $\lim _{m \rightarrow \infty} x_{m}=h$ and $\lim _{m \rightarrow \infty} N_{m}=h$, where $h \in F(T)$, which, $\forall u \in$ $F(T)$, is the unique solution of the variational inequality $\langle(I-k) h, j(h-u)\rangle \leq 0$ and $T$ is asymptotically non-expansive mapping as given in the theorem. It is straightforward to see that as $m \rightarrow \infty$, we get,

$$
\left\|x_{m}-T\left(x_{m}\right)\right\| \leq\left\{1+l_{1}\right\}\left\|x_{m}-h\right\| \rightarrow 0 .
$$

Thus, $\lim _{m \rightarrow \infty}\left\|x_{m}-T\left(x_{m}\right)\right\|=0$. Using the same argument as above, we can show that $\lim _{m \rightarrow \infty}\left\|N_{m}-T\left(N_{m}\right)\right\|=0$.

Next, for any $m \geq 0$, let $\beta_{m}=1$ and $k\left(x_{m}\right)=u$ in (1), where $u \in M$ and $u \neq h$. Then, (1) can be written as

$$
x_{m+1}-T^{m}\left(x_{m}\right)=\alpha_{m}\left(u-T^{m}\left(x_{m}\right)\right) .
$$

Since, $T$ is asymptotically non-expansive and $h \in F(T),\left\|T^{m}\left(x_{m}\right)-T^{m}(h)\right\|=$ $\left\|T^{m}\left(x_{m}\right)-h\right\| \leq l_{m}\left\|x_{m}-h\right\|$. But, $x_{m} \rightarrow h$, (given), which implies that $\left\|T^{m}\left(x_{m}\right)-h\right\| \rightarrow 0$ or $T^{m}\left(x_{m}\right) \rightarrow h$, as $m \rightarrow \infty$. This implies,

$$
\left.\limsup _{m \rightarrow \infty} \alpha_{m} u-T^{m}\left(x_{m}\right)=\limsup _{m \rightarrow \infty} \| x_{m+1}-T^{m}\left(x_{m}\right)\right) \|=0 .
$$

Since, $T^{m}\left(x_{m}\right) \rightarrow h$ and $u \neq h,\left\|u-T^{m}\left(x_{m}\right)\right\| \nrightarrow 0, m \rightarrow \infty$. Thus, for the above to be true, the only possibility is that $\alpha_{m} \rightarrow 0$, as $m \rightarrow \infty$.

Finally, for any $m \geq 0$, let $M=\{x \in E: x \leq 1\}, T=-I$, where $I$ is identity mapping on $M, k=0$ and $\beta_{m}=1, \gamma_{m}=1$. Then, the sequence in (1) can be written as follows.

$$
\begin{aligned}
x_{m+1} & =\left(1-\alpha_{m}\right) T^{m}\left(x_{m}\right)=\left(1-\alpha_{m}\right)\left(-I^{m}\right) x_{m}=\left(-1^{m}\right)\left(1-\alpha_{m}\right) x_{m} \\
& =(-1)^{m+(m-1)}\left(1-\alpha_{m}\right)\left(1-\alpha_{m-1}\right) x_{m-1} \\
& \vdots \\
& =(-1)^{m+(m-1)+\ldots+1}\left(1-\alpha_{m}\right)\left(1-\alpha_{m-1}\right) \cdots\left(1-\alpha_{0}\right) x_{0} .
\end{aligned}
$$

Since, $T=-I$ has a unique fixed point $0 \in M, \lim _{m \rightarrow \infty}\left\|x_{m+1}-0\right\|=$ $\lim _{m \rightarrow \infty}\{-1\}^{\frac{m(m+1)}{2}} \Pi_{j=0}^{m}\left(1-\alpha_{j}\right)\left\|x_{0}\right\|=0$. This implies that $\Pi_{j=0}^{\infty}\left(1-\alpha_{j}\right)\left\|x_{0}\right\|=$ 0 , which means that $\sum_{j=0}^{\infty} \alpha_{j}=\infty$. 
As already mentioned in Remark 2.1. every nonexpansive mapping is a particular case of an asymptotically nonexpansive mapping. This means that Theorem 3.1 is true for any nonexpansive mapping as well. This is specifically interesting because if $T$ is nonexpansive, then, we can remove the boundedness requirement on $M$ in Theorem 3.1 \{for further details see [1] . Again, since the sequence $\left\{l_{m}\right\}$ is a constant-valued sequence 1 's, in this scenario, we get $\eta_{m}=1, \forall m \geq 0$. Hence from Theorem 3.1, we can obtain the following theorem.

Theorem 3.2. Let $E$ be a real Banach space and let the norm on $E$ be uniformly Gâteaux differentiable, possessing uniform normal structure. Let $M \neq \emptyset$ be closed and convex subset of $E$ and let $k: M \rightarrow M$ be a contraction with $\alpha \in(0,1)$ be its contractive constant. Also, let $T: M \rightarrow M$ be a nonexpansive mapping, with $F(T) \neq \emptyset$. Also, assume $\left(t_{m}\right)$ to be a sequence in $(0,1)$ with $\lim _{m \rightarrow \infty} t_{m}=1$. Given any $x_{0} \in M$ and sequences $\left(\alpha_{m}\right),\left(\beta_{m}\right)$ and $\left(\gamma_{m}\right)$ in $[0,1]$, define a sequence $\left(x_{m}\right)$ as follows.

$$
\begin{aligned}
x_{m+1} & =\alpha_{m} k\left(x_{m}\right)+\left(1-\alpha_{m}\right) T\left(y_{m}\right) \\
y_{m} & =\beta_{m} x_{m}+\left(1-\beta_{m}\right) T\left(z_{m}\right) \\
z_{m} & =\gamma_{m} x_{m}+\left(1-\gamma_{m}\right) T\left(x_{m}\right) .
\end{aligned}
$$

Then, for every $m \geq 0, \exists N_{m} \in M$, such that

$$
N_{m}=\left\{1-\frac{t_{m}}{l_{m}}\right\} k\left(x_{m}\right)+\frac{t_{m}}{l_{m}} T\left(N_{m}\right) .
$$

Also, $\left(N_{m}\right)$ and $\left(x_{m}\right)$ are strongly convergent to some $h \in F(T)$ which, $\forall u \in$ $F(T)$, is the unique solution of the variational inequality $\langle(I-k) h, j(h-u)\rangle \leq 0$, if and only if the following conditions hold.

(1) $\lim _{m \rightarrow \infty}\left\|N_{m}-T\left(N_{m}\right)\right\|=0$ and $\lim _{m \rightarrow \infty}\left\|x_{m}-T\left(x_{m}\right)\right\|=0$,

(2) As $m \rightarrow \infty, \alpha_{m} \rightarrow 0$ and $\sum_{m=0}^{\infty} \alpha_{m}=\infty$.

\section{Conclusion}

In this paper, we introduced a new viscosity approximation method. Strong convergence of proposed method is proved under certain assumptions. In uniformly smooth Banach spaces, Theorem 3.2 extends and improves the corresponding results of $\mathrm{Xu}$ [2, which themselves were an extension of results by Moudafi in [25. Theorem 3.1 extends and improves the results presented by Chidume et al. [3], the scheme presented by Shahzad and Udomene [4, the theorem proved by Lim and Xu [1] and the corresponding results in Schu [[17, [18]]. Our result is also a direct extension, as well as, improvement of the work done by Chang et al. [28.

\section{Competing Interests}

The authors declares that they have no competing interests. 


\section{REFERENCES}

1. Lim, T. C., \& Xu, H. K. (1994). Fixed point theorems for asymptotically nonexpansive mappings. Nonlinear Analysis, 22(11), 1345-1356.

2. Xu, H. K. (2004). Viscosity approximation methods for nonexpansive mappings. Journal of Mathematical Analysis and Applications, 298(1), 279-291.

3. Chidume, C., Li, J., \& Udomene, A. (2005). Convergence of paths and approximation of fixed points of asymptotically nonexpansive mappings. Proceedings of the American Mathematical Society, 133(2), 473-480.

4. Shahzad, N., \& Udomene, A. (2006). Fixed point solutions of variational inequalities for asymptotically nonexpansive mappings in Banach spaces. Nonlinear Analysis: Theory, Methods \& Applications, 64(3), 558-567.

5. Youla, D. C. (1987). Mathematical theory of image restoration by the method of convex projections. Image Recovery: Theory and Application, 29-77.

6. Tian, Z., Zarepisheh, M., Jia, X., \& Jiang, S. B. (2013). The fixed-point iteration method for IMRT optimization with truncated dose deposition coefficient matrix. arXiv preprint arXiv:1303.3504.

7. Mann, W. R. (1953). Mean value methods in iteration. Proceedings of the American Mathematical Society, 4(3), 506-510.

8. Genel, A., \& Lindenstrauss, J. (1975). An example concerning fixed points. Israel Journal of Mathematics, 22(1), 81-86.

9. Bauschke, H. H., \& Combettes, P. L. (2001). A weak-to-strong convergence principle for Fejér-monotone methods in Hilbert spaces. Mathematics of operations research, 26(2), 248-264.

10. Nakajo, K., \& Takahashi, W. (2003). Strong convergence theorems for nonexpansive mappings and nonexpansive semigroups. Journal of Mathematical Analysis and Applications, $279(2), 372-379$

11. Matsushita, S. Y., \& Takahashi, W. (2005). A strong convergence theorem for relatively nonexpansive mappings in a Banach space. Journal of Approximation Theory, 134(2), $257-266$.

12. Kim, T. H., \& Xu, H. K. (2006). Strong convergence of modified Mann iterations for asymptotically nonexpansive mappings and semigroups. Nonlinear Analysis: Theory, Methods \& Applications, 64(5), 1140-1152.

13. Martinez-Yanes, C., \& Xu, H. K. (2006). Strong convergence of the CQ method for fixed point iteration processes. Nonlinear Analysis: Theory, Methods \& Applications, 64(11), $2400-2411$.

14. Su, Y., \& Qin, X. (2008). Monotone CQ iteration processes for nonexpansive semigroups and maximal monotone operators. Nonlinear Analysis: Theory, Methods \& Applications, 68(12), 3657-3664.

15. Guan, J., Tang, Y., Ma, P., Xu, Y., \& Su, Y. (2015). Non-convex hybrid algorithm for a family of countable quasi-Lipschitz mappings and application. Fixed Point Theory and Applications, 2015(1), 214.

16. Liu, Y., Zheng, L., Wang, P., \& Zhou, H. (2015). Three kinds of new hybrid projection methods for a finite family of quasi-asymptotically pseudocontractive mappings in Hilbert spaces. Fixed Point Theory and Applications, 2015(1), 118.

17. Schu, J. (1991). Iterative construction of fixed points of asymptotically nonexpansive mappings. Journal of Mathematical Analysis and Applications, 158(2), 407-413.

18. Schu, J. (1991). Approximation of fixed points of asymptotically nonexpansive mappings. Proceedings of the American Mathematical Society, 112(1), 143-151.

19. Bruck, R., Kuczumow, T., \& Reich, S. (1993). Convergence of iterates of asymptotically nonexpansive mappings in Banach spaces with the uniform Opial property. In Colloquium Mathematicae 65(2), 169-179). 
20. Tan, K. K., \& Xu, H. K. (1994). Fixed point iteration processes for asymptotically nonexpansive mappings. Proceedings of the American Mathematical Society, 122(3), 733-739.

21. Shioji, N., \& Takahashi, W. (1999). Strong convergence of averaged approximants for asymptotically nonexpansive mappings in Banach spaces. Journal of Approximation Theory, 97(1), 53-64.

22. Shioji, N., \& Takahashi, W. (1999). A strong convergence theorem for asymptotically nonexpansive mappings in Banach spaces. Archiv der Mathematik, 72(5), 354-359.

23. Kim, T. H., \& Xu, H. K. (2000). Remarks on asymptotically nonexpansive mappings. Nonlinear Analysis: Theory, Methods \& Applications, 41(3-4), 405-415.

24. Chang, S. S. (2001). Some results for asymptotically pseudo-contractive mappings and asymptotically nonexpansive mappings. Proceedings of the American Mathematical Society, 129(3), 845-853.

25. Moudafi, A. (2000). Viscosity approximation methods for fixed-points problems. Journal of Mathematical Analysis and Applications, 241(1), 46-55.

26. Chang, S. S. (2006). Viscosity approximation methods for a finite family of nonexpansive mappings in Banach spaces. Journal of Mathematical Analysis and Applications, 323(2), 1402-1416.

27. Naqvi, S. F. A., \& Khan, M. S. (2017). On the viscosity rule for common fixed points of two nonexpansive mappings in Hilbert spaces. Open J. Math. Sci, 1, 110-125.

28. Chang, S. S., Lee, H. J., Chan, C. K., \& Kim, J. K. (2009). Approximating solutions of variational inequalities for asymptotically nonexpansive mappings. Applied mathematics and computation, 212(1), 51-59.

29. Geobel, K., \& Kirk, W. A. (1990). Topics in metric fixed point theory Cambridge Stud. Adv. Math, 28.

30. Banach, S. (1932). Théorie des opérations linéaires, Monografie Mat., PWN, Warszawa.

31. Liu, L. S. (1995). Ishikawa and Mann iterative process with errors for nonlinear strongly accretive mappings in Banach spaces. Journal of Mathematical Analysis and Applications, 194(1), 114-125.

32. Noor, M. A. (2000). New approximation schemes for general variational inequalities. Journal of Mathematical Analysis and applications, 251(1), 217-229.

33. S. Mazur, J. Schauder, Uber ein prinzip in der variationsrechnung, Proc. Int. Congress Math., Oslo, 1936.

Muhammad Akmal

Department of Mathematics, Lahore Leads University, Lahore, Pakistan.

e-mail: m.akmalwattu@yahoo.com

Muhammad Saqib Khan

Department of Mathematics, Lahore Leads University, Lahore, Pakistan.

e-mail: khan.saqib@me.com

Shahzad Ahmad Maitla

Department of Mathematics, University of Management and Technology, Sialkot, Pakistan.

e-mail: shahzad.ahmed@skt.umt.edu.pk 